\title{
The use of Reverse Rngineering in the Design of New Stamping Dies
}

\author{
Ireneusz Wróbel \\ Department of Fundamentals of Machine Building, University of Bielsko-Biała, ul. Willowa 2, 43-309 Bielsko-Biala, Poland
}

\section{BIOGRAPHICAL NOTES}

Ireneusz Wróbel, Ph.D., works as a lecturer in the Department of Mechanical Engineering Fundamentals at University of Bielsko-Biala. His scientific and didactic work concerns the issues of machine designing and technology, computer aided design, Finite Element Method and reverse engineering. He is the author or co-author of 60 original scientific papers.

\section{KEYWORDS}

Reverse engineering, 3D scanning, Stamping, Drawpiece.

\section{ABSTRACT}

The paper presents a methodology developed by the author for reconstructing surfaces of shaping punches and die blocks. It involves the use of specialised tools available in a commercial CAD system. These are the tools that are used to adjust selected surfaces of the geometricmodel of a part to the cloud of points representing the surface of the part, which may be obtained from a scan. The usefulness of these tools is examined usinga punch used in the automotive industry as an example.

\section{INTRODUCTION}

Stamping is a widely used process of sheet plastic forming. It is performed on mechanical or hydraulic presses with the use of special tools called stamping dies. Stamping (hot stamping or cold stamping) results in a permanent change of shape and dimensions of the processed sheet and is widely used in shaping body and chassis parts. Approximately $95 \%$ of parts in modern car are manufactured using stamping technology. Stamping as a technology for shaping machine parts has many advantages, among which are:

1. Relatively low unit costs,

2. The ability to provide complex shapes,

3. Repeatability of parts,

4. Automation of the production process.

\section{Stamping die Design}

Three-dimensional CAD systems are commonly used for designing stamping dies. The design process is complicated. It requires a lot of experience from the designer and knowledge of metallurgy, sheet metal working and stamping tools (dies) construction. A modern die is a very complex tool. Figure 1 shows a model of a progressive die. This device consisting of a drawing die, sizing tool, blanking tool, clamps, stops.

Stamping die design starts with a three-dimensional CAD model drawpiece. The next step is to develop an operation plan to be carried out by the die. The plan cov- 
ers all the operations (stamping, cutting, sizing, bending), which will be carried out in order to produce drawpieces. At this stage the type of a die is defined(progressive, transfer or one-cut). Based on the plan of operations, parts of the die such as punches, die blocks, clamps and all auxiliary components are designed. The end result of this step is a three-dimensional assembly model of the die and all technical documentation necessary to produce the die. The entire design and evaluation process of the die is shown schematically in Figure 2.

After completing the die parts and their assembly the tool is evaluated. This involves testing under production conditions which verifies the die operation and the shape-dimensional accuracy of the drawpieces. A series of tests and measurements of the drawpieces are performed.

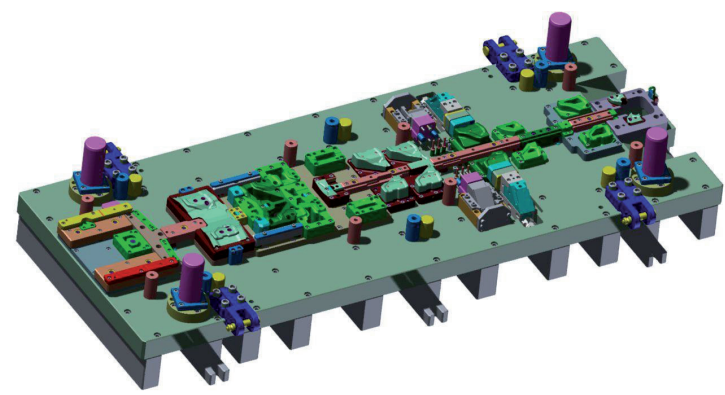

Fig. 1: CAD model of a progressive die.

Frequently test drawpieces do not have the required shape-dimensional accuracy. In this case, it is necessary to introduce corrections to the die blocks and punches. The corrections involve removal and build up of the die blocks. This process of improving the die involves the use of hand tools such as grinding machines and files, and welding equipment. After the corrections has been carried out, further tests are performed. If the drawpiece still does not meet the requirements of shapedimensional accuracy, there follows further improvement of the die which is repeated until the the requirements are met. Often the final die differs significantly from the shape of the die blocks and punches which were designed. Although the die has been modified to give drawpieces with the required shape-dimensional precision, this tool is inconsistent with the developed documentation 3D CAD model of the die.

Tool designers and technologists working in the mills have identified the need to archive the final

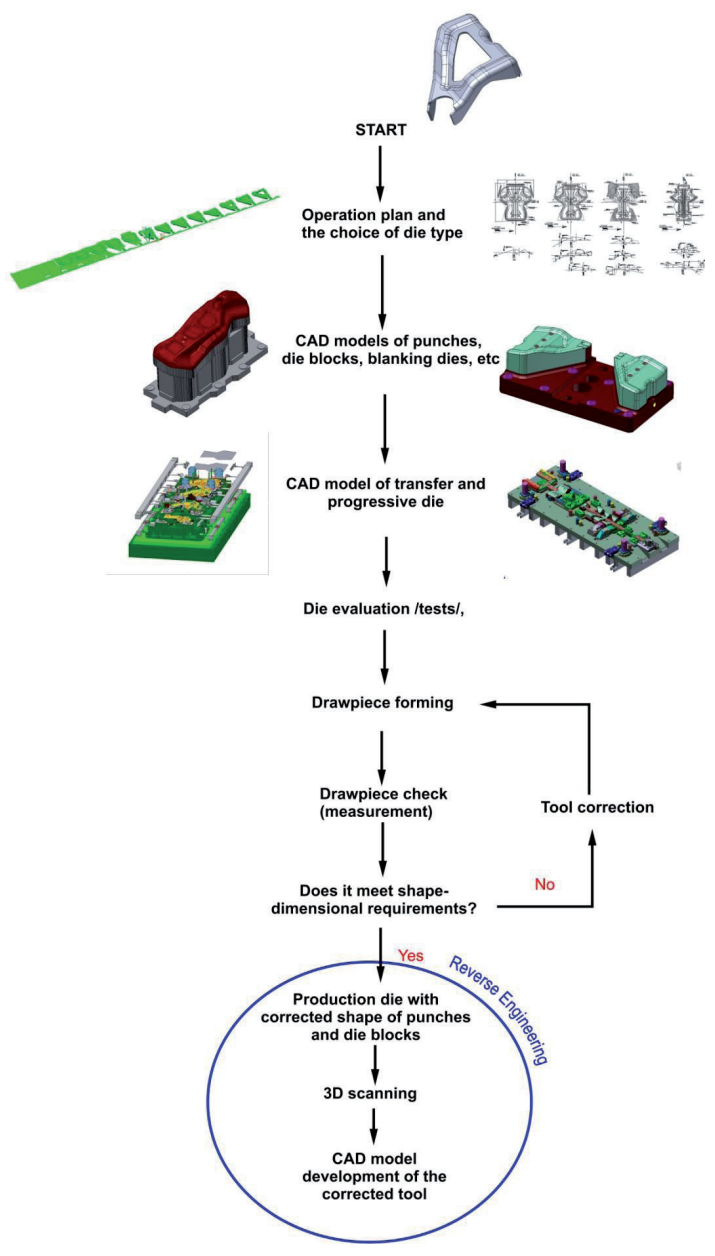

Fig. 2: Die design process.

die shape. To ensure the tool design documentation as a three-dimensional CAD model records the final solution. These records would allow duplicate tools to be produced and assist in the recovery of worn tools.

This article describes the method of measuring the die and using the data from the measurement in the construction of the tool CAD model.

\section{Reverse Engineering in die designing}

The essence of reverse engineering is to reverse the traditional design process where we move from the abstract (virtual CAD model) to the real part - material model. Thus, reversing the process we move from the real physical model which is measured using a 3D scanner and processed to generate a virtual model - 3D CAD model with the use of CAD environment. The remainder of this ar- 
ticle discusses the proposed methodology for using reverse engineering in die design, to precisely reconstruct the die punch shape with the corrections implemented following pre-production testing. The methodology is discussed using the example of a drawing die punch of a progressive die for manufacturing a drawpiece for the automotive industry.

The idea of the proposed methodology is shown in Figure. 3. The procedure consists of several steps, from scanning to checking the accuracy of the obtained solutions.

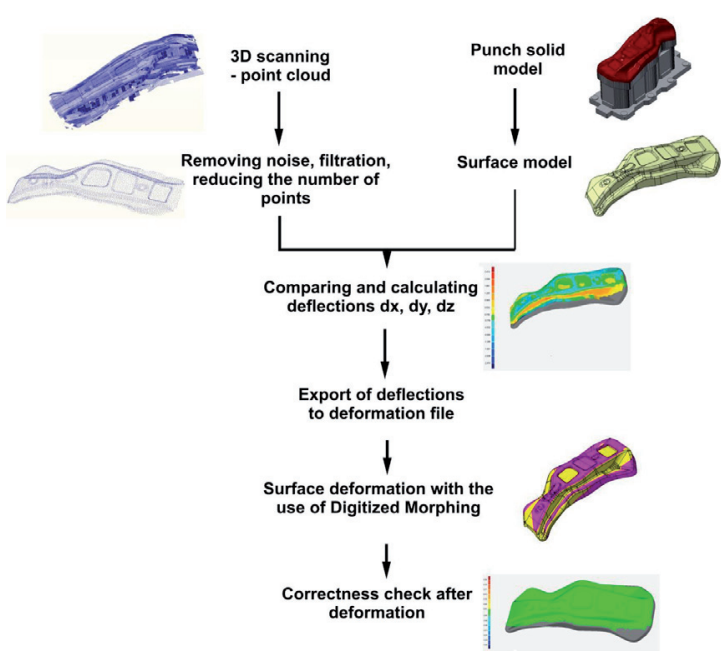

Fig. 3: Methodology of punch reconstruction.

To obtain the CAD model of the punch after reconstruction it is necessary to measure the punch . Due to frequently complex shapes - the measuring device, especially useful for this type of a component is an optical scanner. For the purpose of the presented example, Zeiss LineScan laser scanner is used for the measurement.

The result of punch scanning is a point cloud. This cloud often contains too many points, incorrect points and other noise. In the first step, noise should be filtered out and incorrect and unnecessary points removed. In this case, the point cloud obtained from the scanner has 585000 points. Figure 4 shows a cloud of points obtained after die scanning.

Specialised tools used in systems for reverse engineering, are used to remove noise (which are the result of scanning errors) and the number of points in the cloud is reduced. Noise filtering consists pri-

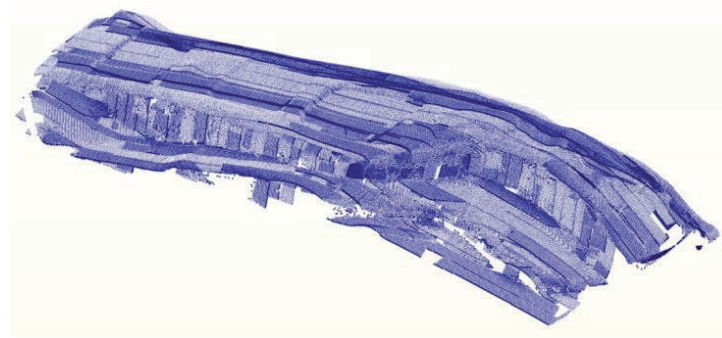

Fig. 4: A point cloud obtained by scanning the die.

marily of the removal of points that stand out from the cloud and the removal of point groups which are not connected with a point in the cloud. To this end, the Leios EDS software is used. Ultimately, a point cloud containing 10000 points is obtained. A series tests carried out by the author show that this is a sufficient number of points for further processing. A point cloud which is too dense, does not increase the accuracy of the operation. Figure 5 shows a rarefied cloud of points used in the further process of the geometry reconstruction.

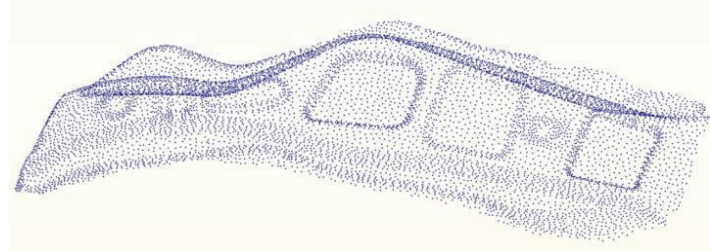

Fig. 5: Point cloud after processing.

The most important step in the punch recovery model is the use of specialised tools available in commercial CAD systems (CATIA), which deforms a selected surface of the geometric model to fit the measured results from the corresponding surface of the real part. Information as to how to deform the selected surface are stored in the deformation file. This file stores coordinates of the points and corresponding displacements in three directions $d x, d y, d z-$ as shown in Figure 6.

The deformation file is generated in two steps. The first step consists of the best adaptation of a point cloud to the surface model of the punch. To complete the best fitting Iterative Closest Point algorithm can be used. In practice, appropriate tools in commercial CAD systems are used. Figure 7 shows the result of fitting the cloud of points to the surface.

In the second stage $\mathrm{dx}$, dy and $\mathrm{dz}$ displacement 


\begin{tabular}{|c|c|c|c|c|c|}
\hline \multicolumn{3}{|c|}{$\begin{array}{l}\text { Coordinates of points from } \\
\text { 3D scanner }\end{array}$} & \multicolumn{3}{|c|}{$\begin{array}{l}\text { Calculated displacement } \\
\text { values in } x, y, z \text { directions }\end{array}$} \\
\hline $\mathbf{x}$ & $y$ & z & $d x$ & dy & $d z$ \\
\hline-58.710720 & -165.153489 & 17.768720 & 0.156987 & -0.055596 & 0.335071 \\
\hline 26.554288 & -475.492030 & 28.441740 & 0.078166 & 0.064343 & -0.190975 \\
\hline-16.813479 & -504.806995 & -40.414352 & -0.791715 & -0.177443 & 0.720900 \\
\hline-93.717299 & -209.360093 & 18.479684 & 0.162654 & 0.008926 & -0.193372 \\
\hline 19.861430 & -30.364487 & 4.765449 & 0.001442 & -0.101272 & -0.513636 \\
\hline-68.645112 & -74.421190 & 11.903611 & 0.003211 & -0.084519 & -0.567696 \\
\hline
\end{tabular}

Fig. 6: Text file format for model deformation.

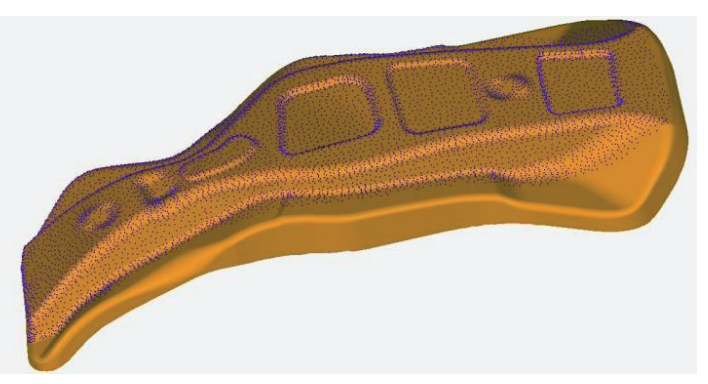

Fig. 7: Point cloud adapted to the surface model.

values should be calculated at each cloud point. To do this, we select any point of the cloud and look for the nearest point on the surface. In this way, point 2 is calculated and the coordinates $d x$, dy and $\mathrm{dz}$ are calculated from the distance between these points. Both steps (fitting and distance calculation) are performed using the Leios system.

The Deformation file is used by Digitising Morphing tool (CATIA) - as shown in Figure 8. These tools are used to build the model of the actual surface of the punch.

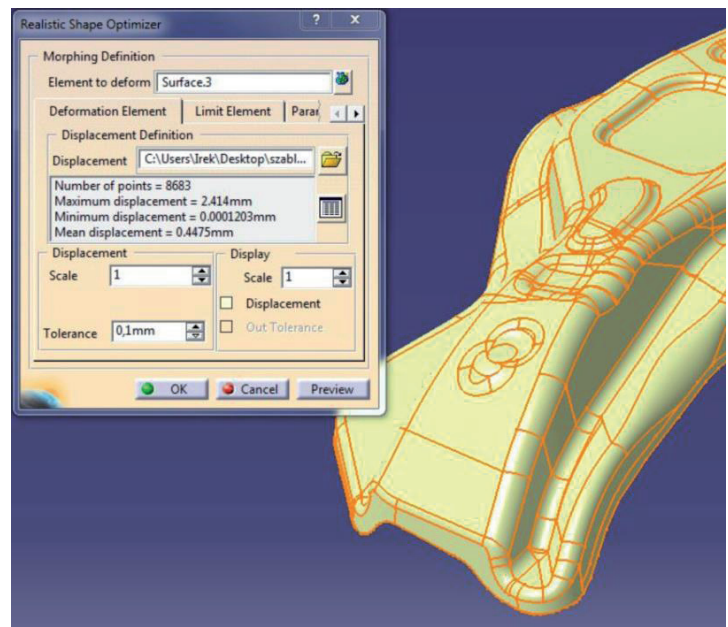

Fig. 8: Tools for deformation of surface models in CATIA.

The result of these operations is a deformed surface which takes the form of a point cloud. Figure 9 shows the result of this tool operation.

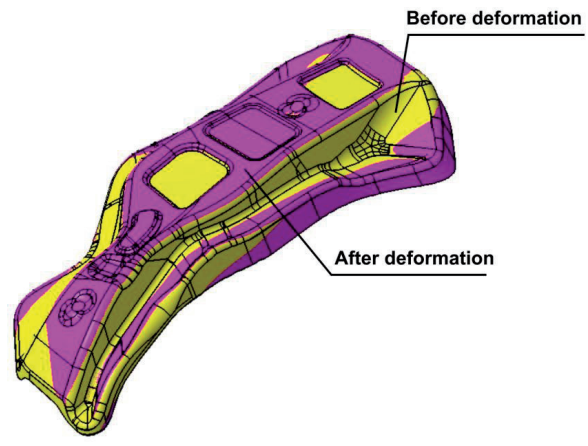

Fig. 9: Surfaces before and after deformation.

In order to validate the accuracy of the performed deformation, it is necessary to compare the surface obtained with the point cloud obtained from the scanner. The result of comparison are distance contour lines measured between the point cloud and the analysed surface. They should be close to zero, which means that the shape of the generated surface corresponds to the shape measured on the real punch. Figure 10 shows the distance contour lines between the deformed surface and the point cloud. This comparison was performed using the Leios system.

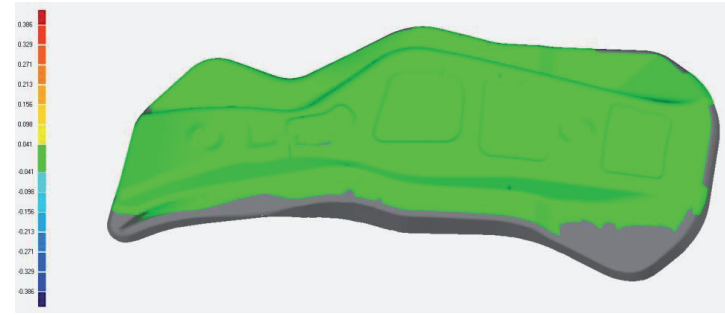

Fig. 10: Distance contours lines between the point cloud and the deformed surface.

The obtained surface can be used for building a solid model of the punch and die block of the designed stamping die. Ultimately this model may be used to produce new punch tools without the need for correction.

\section{Conclusions}

Based on the proposed methodology, the following conclusions can be drawn:

1. The proposed CAD methodology for model reconstruction of the surface shaping of the punch or die block gives good results. It has been implemented by a manufacturer of this type 
of tool.

2. The traditional construction methodology of the three-dimensional CAD model of the punch or die block using reverse engineering technique is very labour intensive.

3. The proposed methodology is many times faster.

4. The resulting models can be used to produce new punch tools without the need for correction or to guide the repair of existing tools.

\section{References}

[1] Wego Wang, CRC Press, 2010, Reverse Engineering - Technology of Reinvention.

[2] Weir, D., Milroy, M., Bradley, C., and Vickers, G., Proc. Inst. Mech. Eng., 1996, Reverse engineering physical models employing wrap-around B-spline surfaces and quadrics.

[3] Help LEIOS Studio.

[4] Kiciak P., WNT, Warszawa 2005, Podstawy modelowania krzywych i powierzchni.

[5] Liang-Chia Chen, Grier C.I. Lin, Robotics and Computer Integrated Manufacturing 16 (2000), Reverse engineering in the design of turbine blades a case study in applying the MAMDP.

[6] Eyup Bagci, Advances in Engineering Software 40 (2009), Reverse engineering applications for recovery of broken or worn parts and remanufacturing: Three case studies.

[7] Besl, P. and McKay, N. , Trans. PAMI, Vol. 14, No. 2, 1992, A Method for Registration of 3-D Shapes

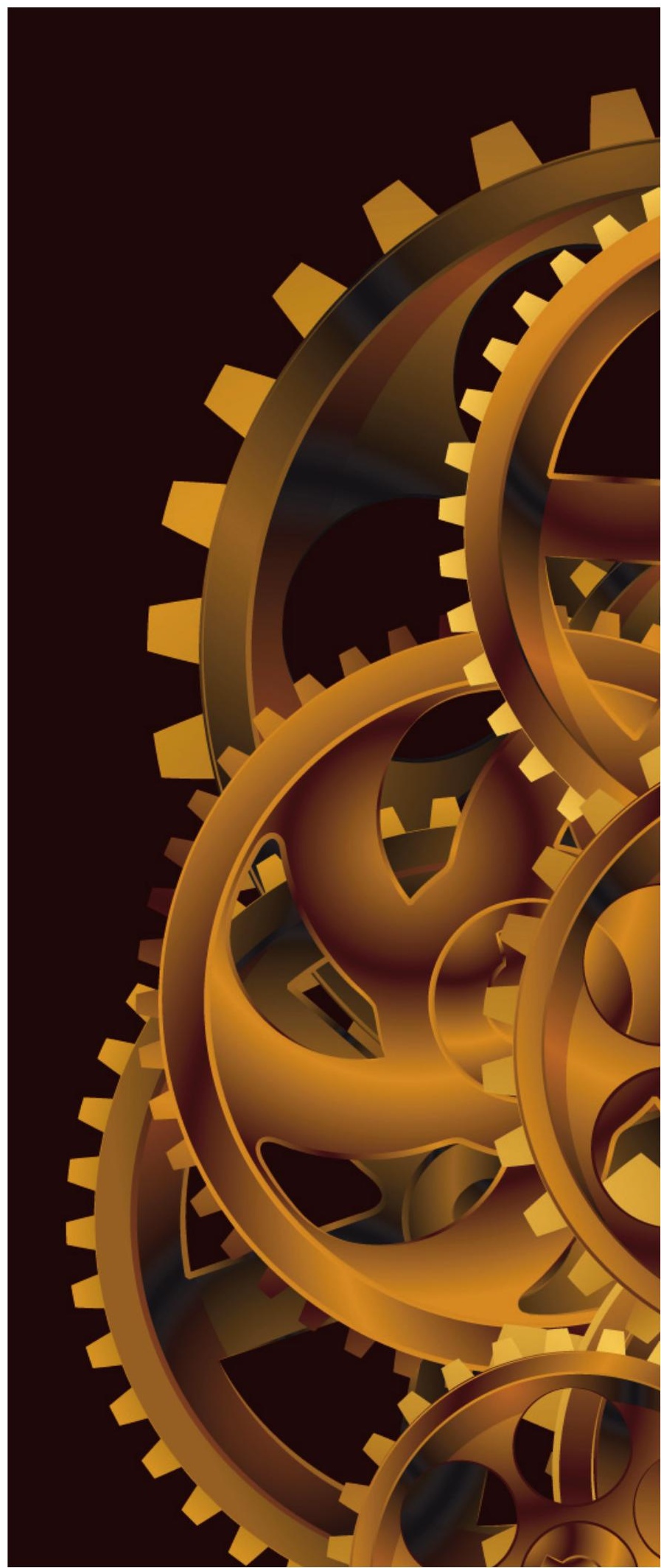




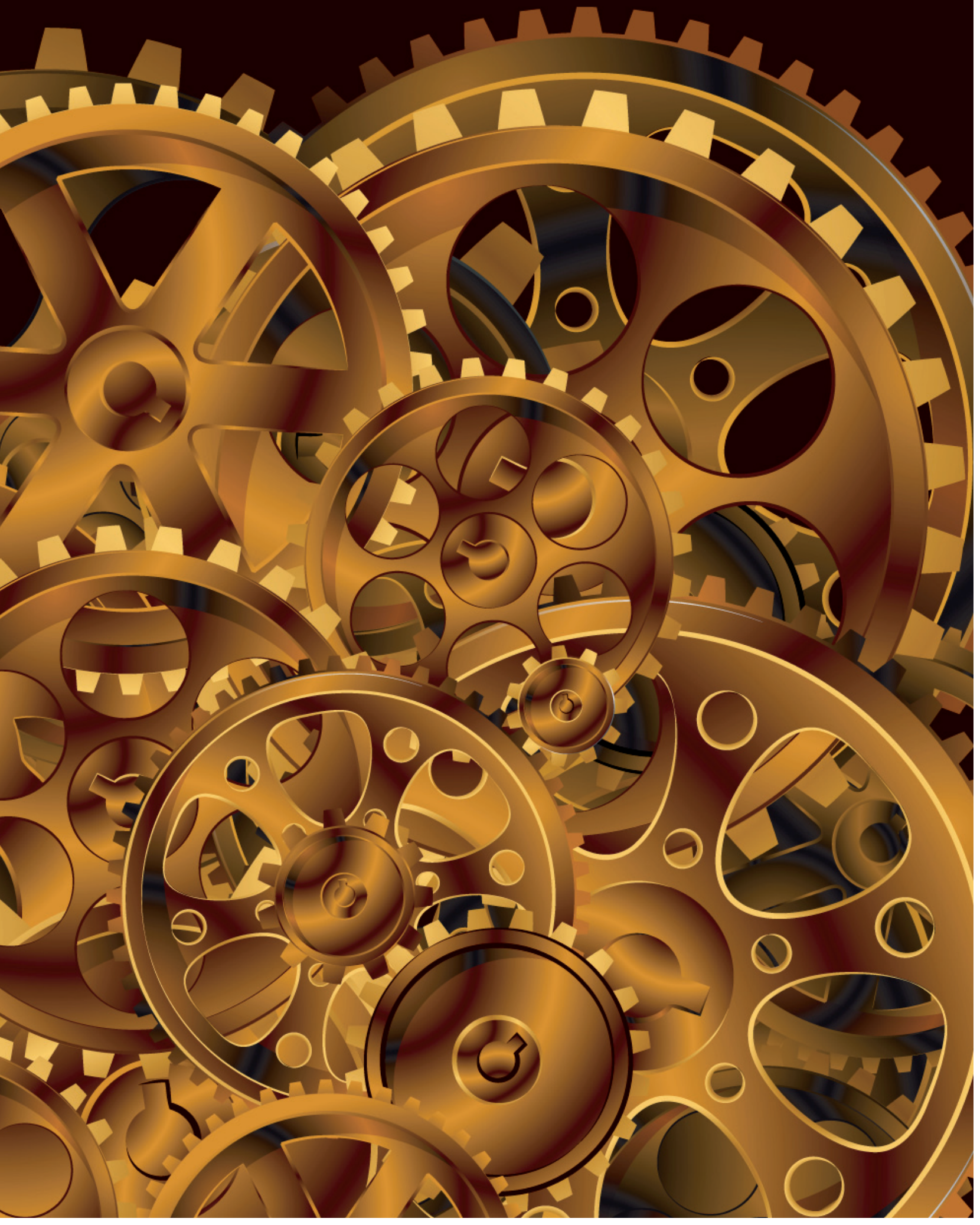

